\title{
ADVANCES IN PRESERVATION OF FRUITS AND VEGETABLES WITH BIOACTIVE COATINGS
}

\author{
ROMEO ROJAS* \\ ANTONIO A. VICENTE** \\ CRISTÓBAL N. AGUILAR***
}

\begin{abstract}
Bioactive compounds are a large group of compounds (antimicrobials, antioxidants, nutrients, etc.), but its use in edible films and coatings for application on fruits and vegetables has been very important because nowadays the consumers demand fruits and vegetables that are fresh, healthy, high quality and easy to prepare. A number of investigations have shown that the use of additives in edible films and coatings improve its functionability and provide compounds for human health. However, it is necessary to continue research that can generate specific or tailor-made edible films and coatings for each product with the best characteristics for preservation. In this review we present and analyze the concepts, progress and perspectives in the design and application of edible films and coatings for fruits and vegetables in order to define the challenges and opportunities that this topic of study in the field of science, technology and food engineering.
\end{abstract}

KEY-WORDS: ADDITIVES, SHELF LIFE, INDUSTRIAL APPLICATIONS, REGULATION, COMMERTIAL MANUFACTURE.

* $\quad$ PhD in Food Science and Technology. Department of Food Science and Technology. School of Chemistry. Universidad Autónoma de Coahuila. Blvd. Venustiano Carranza, 25,000. Saltillo, Coahuila, México. (e-mail: romeorojas@uadec.edu.mx)

** PhD in Chemical and Biological Engineering. Associate Professor with Habilitation. IBB - Institute for Biotechnology and Bioengineering, Centre of Biological Engineering, Universidade do Minho, Campus de Gualtar, 4710-057 Braga, Portugal. (e-mail: avicente@deb.uminho.pt)

*** PhD in Biotechnology. Full Time Professor. Department of Food Research (DIA). School of Chemistry. Universidad Autónoma de Coahuila. Blvd. Venustiano Carranza, 25,000. Saltillo, Coahuila, México. (e-mail: cristobal.aguilar@uadec.edu.mx) 


\section{INTRODUCCIÓN}

Las películas y cubiertas comestibles a base de ceras y/o polisacáridos sobre varios frutos o vegetales han sido usadas por siglos para prevenir la pérdida de humedad mediante la creación de una delgada y continua barrera sobre la superficie del producto. Hoy, los consumidores tienen expectativas muy elevadas en torno a los alimentos ya que deben ser nutritivos, seguros, variados y con larga vida de anaquel. En cuanto a los productos naturales, mantener su vida de anaquel por periodos prolongados es realmente difícil debido a que sufren cambios fisicoquímicos y bioquímicos los cuales afectan su calidad y aceptabilidad (KAYS \& PAULL 2004) y cuando los productos son mínimamente procesados es aún mas difícil. En un principio, estos productos mínimamente procesados solo se ofrecían en restaurantes o eran suministrados por empresas que comercializaban el producto dentro de un periodo muy corto. Ahora, las frutas y hortalizas frescas cortadas se comercializan en supermercados en respuesta a la demanda de los consumidores. Por lo tanto, las frutas y/o verduras mínimamente procesadas se han convertido en un tema de estudio importante para la comunidad científica y tecnológica del área de alimentos en los últimos años.

Para conservar las frutas y hortalizas mínimamente procesadas existen diversos métodos como la refrigeración, el uso de antioxidantes, antimicrobianos, atmosferas controladas, empaques, etc. Sin embargo, existe una vertiente en la conservación de este tipo de productos enfocada al uso de cubiertas y películas comestibles. El propósito de su uso radica en retrasar la transferencia de gases, vapor de agua, compuestos volátiles, cambios en color proporcionando una atmosfera modificada que disminuya la respiración y la senescencia. Sin embargo, el uso de compuestos bioactivos (compuestos antipardeamiento, antimicrobianos, nutrientes, precursores de aromas, antioxidantes, entre otros.) en las películas y recubrimientos han demostrado que potencian la actividad de estos revestimientos. La aplicación de un revestimiento es simple mediante la formación de una capa delgada del material sobre la superficie del alimento mientras que la aplicación de películas implica el pre-fabricado de material. En la presente revisión se presenta el origen, fabricación, y uso de películas y cubiertas comestibles como portadores de compuestos bioactivos para frutas y hortalizas.

\section{MATERIALES Y MÉTODOS}

\subsection{PELÍCULAS Y CUBIERTAS COMESTIBLES}

Se define a estos, como cualquier tipo de material utilizado para envolver un alimento que pueda comerse junto con él se considera película o recubrimiento comestible con un grosor menor a $0,3 \mathrm{~mm}$. Las películas comestibles pueden ser la sustitución y/o enriquecimiento de las capas naturales del producto para evitar pérdida de humedad, proveer un intercambio selectivo a los gases como $\mathrm{O}_{2}, \mathrm{CO}_{2}$ y etileno principalmente, los cuales están implicados en el proceso de respiración. También, pueden proporcionar esterilidad superficial y evitar pérdida de otros componentes volátiles (PAVLATH \& ORTS 2009).

Este tipo de recubrimientos o películas deben estar fabricados a base de materiales reconocidos como seguros (GRAS) en la cantidad a la cual se usan. Regularmente deben estar aprobados por la Food and Drugs Administration (FDA), sin embargo, como es poco práctico tener una lista de todos los ingredientes existe la posibilidad de adquirir el estatus GRAS antes mencionado. Para este caso (GRAS), los fabricantes solicitan la aprobación de un compuesto, ingrediente o alimento siempre y cuando este sustentado con estudios, para lo cual existen 3 tipos de designaciones (Tabla 1): 


\section{Designación}

Definición

Donde el fabricante llevó a cabo todas las investigaciones y paneles de expertos que están dispuestos a defender el estatus de GRAS en caso de impugnación.

Donde los datos han sido presentados a la FDA para su aprobación.

Cuando la FDA ha revisado la reclamación de productos GRAS y dictamina que no existen retos adicionales.

Sin embargo, el estatus de GRAS no garantiza la completa seguridad del producto para personas alérgicas como la intolerancia a la lactosa, celiacos o fenilcetonúricos.

\subsection{FABRICACIÓN}

Para la fabricación de recubrimientos comestibles todos los componentes deben ser GRAS. Esto es un requerimiento importante para elegir los componentes y su concentración. Se pueden utilizar polisacáridos, proteínas, plastificantes (polioles principalmente), lípidos. Además, se debe verificar la compatibilidad de los componentes entre sí y con el producto a recubrir. La concentración de los componentes es esencial debido a que de ello dependerá si el recubrimiento se requiere que sea visible o no perceptible. El proceso de pre-fabricado o laminado de películas comestibles depende principalmente del tipo de componentes que la comprenden y la finalidad de su uso. El uso de ingredientes activos también estará determinado por el uso final del recubrimiento (ROSSMAN 2009).

El procedimiento estará definido por el tipo de componentes a incorporar. Sin embargo, la finalidad es dispersar los componentes en el solvente (regularmente agua), si se trata de una emulsión homogenizar a determinada velocidad de agitación ( $\mathrm{g}$ o rpm), tiempo y temperatura (si se requiere). Pero, se debe considerar que el "scale-up" no es una ciencia exacta y habrá variaciones y detalles que afinar los cuales se deben ir considerando desde la producción nivel planta piloto. Otro factor determinante es el orden de adición de los componentes usando determinado equipo.

Para el proceso de laminado es esencial cuando la finalidad es la fabricación de películas. A nivel laboratorio se puede realizar en recipientes de plástico como cajas Petri y secar por $10-15 \mathrm{~h}$ a $45^{\circ} \mathrm{C}$ con un sistema de aireación. El proceso se complica para la fabricación a nivel industrial cuando el tiempo, espacio, mano de obra, equipo, electricidad, costo de equipos, etc. es muy elevado. Por esta razón, debe llevarse a cabo en equipos especializados diseñados específicamente para este fin. Algunas metodologías que pueden ser utilizadas puede ser hornos o cámaras de secado, trasportadores de cinta de acero con las cuales se puede remover el excedente de humedad y formar el laminado, las cuales en Estados Unidos se fabrican por Berndorf Belt Systems (Elgin, IL) y Sandvik Process Systems, Inc. (Totowa, NJ). Otro proceso que puede ser utilizado son las Ilamadas líneas de recubrimiento las cuales permiten una excelente estabilidad dimensional y un mínimo de acumulación de electricidad estática en el proceso (ésta la han fabricado la empresa Radiant Energy Systems, Hawthorne, NJ a nivel piloto, y a nivel industrial Faustel, Inc., Germantown, WI). Otro método alternativo es la extrusión en estado fundido, sin embargo, un exceso de 
plastificante puede producir migración a la superficie de la película, espuma en la película, condensación en el área de secado y la temperatura de secado (hasta $200{ }^{\circ} \mathrm{C}$ o más) puede provocar degradación de los componentes (ROSSMAN 2009).

\subsection{BENEFICIOS DE LAAPLICACIÓN DE PELÍCULAS Y/O RECUBRIMIENTOS COMESTIBLES}

Actualmente, alrededor del mundo el método de conservación más empleado para alimentos ligeramente procesados es la refrigeración $\left(4-8{ }^{\circ} \mathrm{C}\right)$. El efecto de la refrigeración radica en la disminución de las actividades enzimáticas deteriorantes aunque esta temperatura también puede ocasionar aumentos en la producción de etileno (hormona de la maduración) (EAKS \& LUDI 1960). Otra forma de conservación es usar temperaturas congelantes (por debajo de $0{ }^{\circ} \mathrm{C}$ ) para inhibir el crecimiento de hongos, sin embargo, a estas temperaturas no se eliminan las reacciones fisicoquímicas (DONHOWE \& FENNEMA 1993) y en algunos frutos tropicales estas temperaturas causan daño en la membrana celular sin dejar de lado existen microorganismos psicrófilos.

Otra de las tecnologías de conservación para frutas y vegetales frescos son las atmósferas controladas que básicamente consisten en controlar la transferencia de los gases dentro y fuera del producto a fin de prolongar su vida de anaquel mediante el uso de gases a temperaturas óptimas. Esta combinación de gases es capaza de ralentizar el metabolismo respiratorio y por ende conservar la calidad fisicoquímica del producto. Sin embargo, es una tecnología cara y se deben tener muchos cuidados como mantener la temperatura adecuada para evitar condensación de vapor de agua y promover la descomposición (PAVLATH \& ORTS 2009).

En respuesta a esta gran demanda a nivel mundial, algunos grupos de investigación de varios países del mundo se han centrado en proveer alternativas de empacado para casi todo tipo de alimentos. Toda esta búsqueda se ha llevado a cabo en los últimos 50 años debido a que cada producto es diferente y debe ser empacado bajo condiciones específicas para lograr mantener la degradación a niveles naturales o reducirla. Sin embargo, no toda la investigación se ha centrado en prolongar vida de anaquel, sino también en recubrimientos de presentación como para manzanas, dulces, píldoras (para evitar que se desmorone, que se perciba su sabor amargo o para brindar una liberación prolongada). En la fabricación de papas fritas también se utiliza como controlador de perdida de humedad y reducir la absorción de aceite (FEENEY et al., 1992; POLANSKI 1993).

Las frutas y vegetales frescas mantienen su respiración y una determinada cantidad de agua que se mantiene cuando se encuentran en su entorno natural. Cuando este delicado entorno se modifica al disminiurse la actividad de agua y las reacciones fisiológicas, entre muchos otros factores, hacen que el proceso de descomposición se acelere. El entorno de $\mathrm{O}_{2}$ y $\mathrm{CO}_{2}$ se modifica, la producción de etileno (si es el caso) se acelera, cambiando el color, sabor, textura, y contenido nutrimental (WONG et al., 1994). El uso de tecnologías como atmosferas controladas, bajas temperaturas durante el transporte reducen el proceso de senescencia (por ejemplo, atmosfera modificada con HR alta, reduce la pérdida de humedad). Sin embargo, con todos los intentos de entornos controlados intentando simular las condiciones naturales no han sido suficientes y además el costo de estas tecnologías es algo desalentador.

Por otro lado, este tipo de problemas no se limita a las frutas y vegetales. La carne, pasteles y confitería también se deterioran antes de llegar al consumidor debido a su alto contenido en proteínas, sabor, lípidos, vitaminas, etc. Pero también ha habido respuesta a este tipo de demandas como una patente que sirve para cubrir carne con gelatina para retrasar el desarrollo de microorganismos y 
pérdida de agua (HAVARD \& HARMONY 1869). También, la aplicación de recubrimientos a base de polisacáridos ha logrado retardar la pérdida de agua en productos cárnicos (ALLEN et al., 1963; SHAW et al., 1980). Para carne de res, cerdo, cordero y aves de corral se ha utilizado con éxito el uso de recubrimientos para reducir el crecimiento microbiano (LAZARUS et al., 1976; WILLIAMS et al., 1978). Cuando se encuentran combinados la repostería y la carne (empanadas de carne) el reto es más grande debido a la gran absorción de aceite, pero con el uso de recubrimientos a base de derivados de celulosa este fenómeno se reduce (FEENEY, HARARALAMPU et al., 1992; POLANSKI 1993). Para la confitería, el aceite en el chocolate genera problemas de viscosidad, absorción de humedad y oxidación (NELSON \& FENNEMA 1991; PAULINKA 1986). Para el caso de las gomas de mascar, un recubrimiento comestible es necesario para evitar la pérdida de humedad (MEYERS 1994). Y finalmente, las frutas deshidratadas consideradas "snaks" tienen mayor vida útil cuando se recubren con zeína (COSLER 1957).

La migración de agua (ganancia o pérdida) en el área de los alimentos es considerada como indeseable ya sea en el transporte, almacenamiento o distribución. Incluso si el producto no se deteriora, la pérdida de agua significa pérdida de peso y por ende, pérdida económica. Otra causa de deterioro es el causado por la enzima polifenol oxidasa (oscurecimiento enzimático). Este deterioro no afecta el peso, sin embargo las pérdidas radican en el rechazo de los consumidores. Una solución obvia es protegiéndolos hasta que estén listos para su consumo. Además, el uso de recubrimientos comestibles ofrecen una serie de ventajas, previenen de contaminación microbiana y deterioro postcosecha. También, pueden resolver problemas como las cuarentenas que regularmente se usan en frutas debido a una posible infestación con huevos de insectos. Por ejemplo, cuando los frutos deben ser almacenados a temperaturas de $1-2{ }^{\circ} \mathrm{C}$ antes de la entrega (de Florida-Japón) (ISMAIL 1989). El uso de recubrimientos puede reducir los niveles de $\mathrm{O}_{2}$ y matar la larva (HALLMAN et al., 1994). Sin embargo, la mejor opción para evitar pérdidas de calidad es eliminar la piel del fruto y aplicar recubrimientos hechos a la medida.

\subsection{REGULACIÓN}

Los principales ingredientes de las películas y/o cubiertas comestibles (biopolímeros) por ley son considerados como aditivos. Aunque también pueden llevar componentes adicionales que mejoren sus propiedades, tales como plastificantes, emulsificantes, compuestos funcionales, etc. Sin embargo, la función principal es actuar como barrera. Desde el siglo XVII se ha reportado la aplicación de recubrimientos pero hasta 1942 hay registro de patentes sobre películas comestibles para consumo humano. Regularmente se usan para prolongar la vida de anaquel del producto y así mantener la frescura, conservar o mejorar la apariencia. La FDA define como aditivo alimentario "cualquier sustancia cuyo uso previsto de resultados o que razonablemente se puede esperar que resulte, directa o indirectamente, en su devenir un componente o que afecte las características de cualquier alimento" y a un antimicrobiano como "un compuesto o sustancia que mata microorganismos, evita o inhibe su crecimiento y reproducción en un concentración suficiente para prevenir el deterioro o prevenir el crecimiento de microorganismos añadidos inadvertidamente". Este tipo de antimicrobianos pueden ser naturales, sintéticos, o semi-sintéticos.

\subsection{CUBIERTAS Y PELÍCULAS COMESTIBLES PARA FRUTAS Y VEGETALES}

Las frutas y vegetales son tejidos vivos en los cuales se presentan cambios fisiológicos y bioquímicos que deterioran la calidad y la vida útil de los productos después de la cosecha. La respiración, transpiración y producción de etileno son los principales factores que contribuyen al 
deterioro de las frutas y vegetales (DEELL et al., 2003). Después de su cosecha también se puede presentar un estrés biótico y abiótico que modifica sus propiedades antes de su consumo. Sin embargo, la calidad y vida de anaquel del producto también dependerá fuertemente dependiendo la variedad y el estado de madurez (OLIVAS \& BARBOSA-CÁNOVAS 2005).

La tecnología de recubrimientos comestibles sobre frutas y vegetales no es nueva. HARDENBURG (1967) menciona el uso de cera sobre naranjas y limones para proveer una cubierta capas de prolongar su vida de anaquel en China remontándose al siglo XII. La primera patente en Estados Unidos de Norte América acerca de recubrimientos comestibles para frutas enteras con cera fundida es de Hoffman en 1916 (HOFFMAN 1916). En 1972, Bryan patentó un método para preservar mitades de pomelo con una cubierta de pectina de bajo metoxilo y goma de algarrobo usando zumo de pomelo como solvente. A través del tiempo han existido diferentes opiniones sobre el tema de recubrimientos comestibles para frutas y vegetales (ALI et al., 2010; BALDWIN 1994; BANKS et al., 1993; CLAYPOOL 2004; CURTIS 1988; GONZÁLEZ-AGUILAR et al., 2007; HAGENMAIER 2000; HAGENMAIER \& SHAW 1992; HARDENBURG 1967; MURATORE et al., 2005; OCHOA et al., 2011; PARK 1999; PARK \& CHINNAN 1990; PLATENIUS 1939; SAUCEDOPOMPA et al., 2007; SAUCEDO-POMPA et al., 2009) y frutas y vegetales mínimamente procesados (BALDWIN, E.A. et al., 1995; BALDWIN, E.A. et al., 1995; CHIUMARELLI et al., 2011; LIN \& ZHAO 2007; NISPEROS \& BALDWIN 1996; OLIVAS \& BARBOSA-CÁNOVAS 2005; SAUCEDO-POMPA, JASSO-CANTÚ et al., 2007; SOTHORNVIT \& RODSAMRAN 2008; WONG, CAMIRAND et al., 1994 ) las cuales han abordado el tema indicando diferentes perspectivas.

\subsection{PELÍCULAS Y CUBIERTAS COMESTIBLES ADICIONADAS CON COMPUESTOS BIOACTIVOS PARA FRUTAS Y VEGETALES}

La integración de compuestos bioactivos dentro de una cubierta o película comestible se utiliza dependiendo del objetivo final del recubrimiento, la naturaleza del alimento y la aplicación específica. Los niveles de inclusión de los aditivos varían dependiendo el uso final. Para alimentos nutritivos en los cuales la intención es maximizar el ingrediente activo se puede adicionar el $100 \%$ o más, según los sólidos del polímero. Para fármacos, la cantidad de activo debe ser igual a una dosis precisa por cada unidad de película en los cuales los niveles típicos son entre el $5-20 \%$ en base al polímero (ROSSMAN 2009). Un ejemplo de aplicación en productos alimenticios es en cítricos en donde se ha adicionado agentes antimicrobianos y fungicidas en emulsiones. Diversos autores han reportado muy buenos resultados en la aplicación de imazalil en naranjas valencia (RADINA \& ECKERT 1988), ácido sorbico, tiabendazol y benomilo en toronja; 2, 6-dicloro-4-nitroanilina (DCNA) en duraznos y nectarinas (WELLS 1971); tiabendazol en papaya (Couey y Farias, 1979); iprodine y quitosán en fresas (GHAOUTH et al., 1991) así como captan y tiran en tomates (DOMENICO et al., 1972). En lechuga se ha usado 1-metilciclopropeno y reduce la senescencia, pero el uso de alginato la mantiene crujiente lo que crea la posibilidad de usarlo combinado (TAY \& PERERA 2004). También un recubrimiento de zeína además de ser una buena barrera, reduce la población de Listeria monocytogenes en maíz dulce (CARLIN et al., 2001).

Otro ejemplo es el uso de recubrimientos a base de proteína de suero de leche con glicerol como plastificante, lecitina como tensoactivo, metil parabeno como antimicrobiano o vitamina $\mathrm{E}$ como antioxidantes sobre cacahuate demostraron que las muestras recubiertas se oxidaron más lento que el control (LEE \& KROCHTA 2002). En guayaba, la eficiencia del embalaje depende de la retención de elementos hidrófobos más que vapor de agua (QUEZADA-GALLO et al., 2005). Sin embargo, en los últimos 20 años las películas y cubiertas comestibles con adición de compuestos bioactivos han sido estudiadas usando diferentes combinaciones (Ver tabla 2 y 3 ). 


\section{TABLA 2. RECUBRIMIENTOS COMESTIBLES CON COMPUESTOS BIOACTIVOS APLICADOS EN FRUTAS Y VEGETALES MÍNIMAMENTE PROCESADOS}

\begin{tabular}{|c|c|c|c|c|c|}
\hline Frutas & Componentes & $\begin{array}{l}\text { Compuestos } \\
\text { bioactivos }\end{array}$ & $\begin{array}{l}\text { Variables } \\
\text { evaluadas }\end{array}$ & Resultados & Referencias \\
\hline Mango & $\begin{array}{l}\text { Almidón o alginato } \\
\text { de sodio }\end{array}$ & Ácido cítrico & WL, C & $\begin{array}{l}\text { El recubrimiento } \\
\text { de almidón reduce } \\
\text { la WL y mantiene } \\
\text { el C. El uso de } \\
\text { ácido cítrico es } \\
\text { más eficiente para } \\
\text { conservar el C. }\end{array}$ & $\begin{array}{l}\text { CHIUMARELLI, } \\
\text { FERRARI et al., } \\
2011\end{array}$ \\
\hline Mango & Puré de mango & -- & $\begin{array}{l}\text { WL, C, SS, ST, } \\
\text { Et, F. }\end{array}$ & $\begin{array}{l}\text { Redujo la WL y } \\
\text { prolongó la vida de } \\
\text { anaquel }\end{array}$ & $\begin{array}{l}\text { SOTHORNVIT } \\
\text { \& RODSAMRAN } \\
2008\end{array}$ \\
\hline $\begin{array}{l}\text { Manzana, } \\
\text { plátano y } \\
\text { rebanadas de } \\
\text { aguacate }\end{array}$ & Goma arabiga & Ácido elágico & & $\begin{array}{l}\text { Las cubiertas a } \\
\text { base de goma } \\
\text { arábiga extienden } \\
\text { la vida de anaquel } \\
\text { y retardan el } \\
\text { oscurecimiento. }\end{array}$ & $\begin{array}{l}\text { SAUCEDO- } \\
\text { POMPA, JASSO- } \\
\text { CANTÚ et al., } \\
2007\end{array}$ \\
\hline $\begin{array}{l}\text { Rebanadas } \\
\text { de manzana } \\
\text { (Gala) }\end{array}$ & $\begin{array}{l}\mathrm{CaCl}_{2}+\text { alginato } \\
\text { o alginato/ácido } \\
\text { linoleico }\end{array}$ & $\begin{array}{l}\text { Sorbato de } \\
\text { potasio, alfa- } \\
\text { monoestearato. }\end{array}$ & $\begin{array}{l}\text { WL, C, F, MG, } \\
\text { TA, SS, FV }\end{array}$ & $\begin{array}{l}\text { Todas las cubiertas } \\
\text { conservan la F } \\
\text { y C. } \\
\text { WL fue retrasada, } \\
\text { principalmente con } \\
\text { la cubierta Alg- } \\
\text { ác. linoleic. Altas } \\
\text { cantidades de } \\
\text { butanol, hexanal, } \\
\text { 1-hexanol, trans- } \\
\text { 2-hexenal sobre } \\
\text { las muestras } \\
\text { recubiertas } \\
\text { al final del } \\
\text { almacenamiento. }\end{array}$ & $\begin{array}{l}\text { OLIVAS et al., } \\
2007\end{array}$ \\
\hline $\begin{array}{l}\text { Nuez sin } \\
\text { cáscara }\end{array}$ & $\mathrm{CMC}$ & $\begin{array}{l}\text { PG, Sorbitol, } \\
\text { lecitina, } \\
\text { a-tocoferol, BHA, } \\
\text { BHT, ácido cítrico. }\end{array}$ & ST, R, C & $\begin{array}{l}\text { Mejoro el brillo, la } \\
\text { F no se conserva. }\end{array}$ & $\begin{array}{l}\text { BALDWIN \& } \\
\text { WOOD } 2006\end{array}$ \\
\hline $\begin{array}{l}\text { Rebanadas de } \\
\text { manzana }\end{array}$ & $\begin{array}{l}\text { WPC + Cera de } \\
\text { abeja }\end{array}$ & $\begin{array}{l}\text { AA, cisteína, o } \\
\text { 4-hexylresocinol }\end{array}$ & WL, C, BI, ST & $\begin{array}{l}\text { Las cubiertas } \\
\text { WPC-AA y WPC- } \\
\text { CA reducen B. WL } \\
\text { no se previene } \\
\text { con el uso del } \\
\text { recubrimiento. } \\
\end{array}$ & $\begin{array}{l}\text { PÉREZ-GAGO et } \\
\text { al., } 2006\end{array}$ \\
\hline $\begin{array}{l}\text { Rebanadas de } \\
\text { pera }\end{array}$ & $\begin{array}{l}\text { Metilcelulosa } \\
\text { (MC) y MC-ácido } \\
\text { estearico (SA) }\end{array}$ & $\begin{array}{l}\text { AA, sorbato de } \\
\text { potasio, } \mathrm{CaCl}_{2}\end{array}$ & $\begin{array}{l}\text { WL, F, MG, C, } \\
\text { TA, SS, FV }\end{array}$ & $\begin{array}{l}\text { Los recubrimientos } \\
\text { retardan B. WL se } \\
\text { retrasó en peras } \\
\text { cubiertas con } \\
\text { MC-SA. Peras } \\
\text { recubiertas con } \\
\text { MC-SA contienen } \\
\text { más alta cantidad } \\
\text { de acetato de } \\
\text { hexilo. }\end{array}$ & $\begin{array}{l}\text { OLIVAS et al., } \\
2003\end{array}$ \\
\hline $\begin{array}{l}\text { Zanahorias } \\
\text { pequeñas }\end{array}$ & Goma xantana & $\begin{array}{l}\text { Lactato de calcio, } \\
\text { gluconato de } \\
\text { calcio, vitamina E. }\end{array}$ & $\begin{array}{l}\text { C, ST, Vitamina } \\
\text { E, } \beta \text {-caroteno, } \\
\text { calcio }\end{array}$ & $\begin{array}{l}\text { El contenido de } \\
\text { calcio y vitamina } \\
\text { E se incrementa } \\
\text { en las zanahorias } \\
\text { recubiertas. Las } \\
\text { cubiertas mejoran } \\
\text { C. }\end{array}$ & MEl et al., 2002 \\
\hline
\end{tabular}

Donde: $F$ firmeza; $W L$ pérdida de peso; TA acidez titulable; $S S$ solidos solubles; $A A$ ácido ascórbico; $C$ color; $M G$ crecimiento microbiano; FV compuestos volátiles; $S T$ evaluación sensorial; $G$ brillo; $B$ Índice de oscurecimiento; $B$ oscurecimiento. 


\section{TABLA 3. CUBIERTAS COMESTIBLES CON COMPUESTOS BIOACTIVOS SOBRE FRUTAS Y VEGETALES ENTEROS.}

\begin{tabular}{|c|c|c|c|c|c|}
\hline Frutas & Componentes & $\begin{array}{l}\text { Compuestos } \\
\text { bioactivos }\end{array}$ & $\begin{array}{l}\text { Variables } \\
\text { evaluadas }\end{array}$ & Resultados & Referencias \\
\hline Manzanas & $\begin{array}{l}\text { Goma arábiga/cera } \\
\text { candelilla }\end{array}$ & Ácido elágico & $\begin{array}{l}\text { WL, F, MG, DS, } \\
\text { SS }\end{array}$ & $\begin{array}{l}\text { El uso de cubierta } \\
\text { reduce la WL y el } \\
\text { daño por MG. Se } \\
\text { mantiene la F y la } \\
\text { DS e incrementa } \\
\text { SS. }\end{array}$ & $\begin{array}{l}\text { OCHOA, } \\
\text { SAUCEDO- } \\
\text { POMPA et al., } \\
2011\end{array}$ \\
\hline Aguacate & Goma arábiga & Ácido elágico & $\begin{array}{l}\text { WL, F, MG, DS, } \\
\text { SS }\end{array}$ & $\begin{array}{l}\text { Prolonga la } \\
\text { vida de anaquel } \\
\text { y mantienen } \\
\text { la calidad } \\
\text { microbiológica. }\end{array}$ & $\begin{array}{l}\text { SAUCEDO- } \\
\text { POMPA, } \\
\text { ROJAS- } \\
\text { MOLINA et al., } \\
2009\end{array}$ \\
\hline Fresa & Almidón & Sorbitol & $\begin{array}{l}\text { C, F, WL, SS, } \\
\text { MG, }\end{array}$ & $\begin{array}{l}\text { Recubrimiento } \\
\text { opaco }\end{array}$ & $\begin{array}{l}\text { RIBEIRO et } \\
\text { al., } 2007\end{array}$ \\
\hline Fresa & Quitosán & $\begin{array}{l}\text { Tween } 80 \text {, ácido } \\
\text { oleico }\end{array}$ & $\begin{array}{l}\text { SSD, WVR, } \\
\text { TA, pH, SS, } \\
\text { anthocyanin, } \\
\text { Fungal decay, } \\
\text { RR, MP, ST }\end{array}$ & $\begin{array}{l}\text { El recubrimiento no } \\
\text { tuvo efecto sobre } \\
\text { TA, pH y contenido } \\
\text { de SS. Los frutos } \\
\text { recubiertos } \\
\text { disminuyeron en } \\
\text { aroma y sabor, } \\
\text { cuando la relación } \\
\text { ácido oleico:quitsán } \\
\text { fue alta. }\end{array}$ & $\begin{array}{l}\text { VARGAS et } \\
\text { al., } 2006\end{array}$ \\
\hline $\begin{array}{l}\text { Albaricoques } \\
\text { y pimiento } \\
\text { morrón verde }\end{array}$ & MC + ácido esteárico & $\begin{array}{l}\text { PEG, PEG + CA, } \\
\text { PEG + AA }\end{array}$ & WL, Vitamina C & $\begin{array}{l}\text { El recubrimiento } \\
\text { retraso WL y } \\
\text { se disminuyó la } \\
\text { pérdida de vitamina } \\
\text { C con AA y CA. }\end{array}$ & $\begin{array}{l}\text { AYRANCY \& } \\
\text { TUNC } 2004\end{array}$ \\
\hline Cereza & $\begin{array}{l}\text { Caseinato de sodio } \\
\text { o concentrado de } \\
\text { proteína de leche. }\end{array}$ & $\begin{array}{l}\text { Glycerol y cera } \\
\text { de abejas o ácido } \\
\text { palmítico/estearico }\end{array}$ & $\mathrm{SS}, \mathrm{TA}, \mathrm{pH}, \mathrm{WL}$ & $\begin{array}{l}\text { Recubrimientos } \\
\text { disminuyeron la } \\
\text { WL y tuvieron } \\
\text { efecto benéfico } \\
\text { en la calidad } \\
\text { sensorial. }\end{array}$ & $\begin{array}{l}\text { CERTEL et al., } \\
2004\end{array}$ \\
\hline Ciruela & $\begin{array}{l}\text { HPMC + cera de } \\
\text { abejas o goma laca. }\end{array}$ & Ácido esteárico & WL, F, Et, EtOH & $\begin{array}{l}\text { Disminuyo la WL } \\
\text { a medida que } \\
\text { se incrementó } \\
\text { el contenido de } \\
\text { lípidos. F no se } \\
\text { afectó después } \\
\text { de almacenarlo } \\
\text { a } 20^{\circ} \mathrm{C} \text { por } \\
\text { corto periodo. } \\
\text { Imacenamiento } \\
\text { prolongado redujo } \\
\text { la pérdida de } \mathrm{F} \text {. }\end{array}$ & $\begin{array}{l}\text { PÉREZ-GAGO } \\
\text { et al., } 2003\end{array}$ \\
\hline Mango & $\begin{array}{l}\text { Celulosa. Nature Seal } \\
\circledR 2020 \text { hidroxipropil } \\
\text { metilcelulosa } \\
\text { Carnauba. Tropical } \\
\text { Fruit Coating } 213 \\
\text { (TFC) }\end{array}$ & $\begin{array}{l}\text { Conservador, } \\
\text { acidulante y } \\
\text { emulsificante. } \\
\text { jabón de ácidos } \\
\text { grasos. }\end{array}$ & $\begin{array}{l}\text { IGC, WL, FV, SS, } \\
\text { TA, DS, ST, F }\end{array}$ & $\begin{array}{l}\text { Ambos } \\
\text { recubrimientos } \\
\text { reducen el } \\
\text { deterioro y mejoran } \\
\text { apariencia. Con } \\
\text { polisacárido retraso } \\
\text { la maduración } \\
\text { y aumento la } \\
\text { concentración } \\
\text { de compuestos } \\
\text { volátiles. La } \\
\text { carnauba reduce } \\
\text { la WL. }\end{array}$ & $\begin{array}{l}\text { BALDWIN et } \\
\text { al., } 1999\end{array}$ \\
\hline
\end{tabular}

Donde: $F$ firmeza; $W L$ pérdida de peso; TA acidez titulable; SS solidos solubles; $A A$ ácido ascórbico; $C$ color; $M G$ crecimiento microbiano; IGC Composición interna de gas; FV compuestos volátiles; $D S$ síntomas de deterioro; $S T$ evaluación sensorial; $E t O H$ etanol; Et etileno; SSD Densidad de superficie solida; $W V R$ resistencia al vapor de agua. 
Puntualmente, el uso de agentes antimicrobianos como el ácido benzoico, sórbico, propiónico, sorbato de potasio y/o benzoato de sodio representan una alternativa de conservación de alimentos. Actualmente, se ha estudiado a los recubrimientos como portadores de aditivos debido a que han mostrado efectividad en la retención de aditivos en la superficie de los alimentos. En los últimos años, diversos grupos han evaluado la difusividad del ácido sórbico y el sorbato de potasio en sistemas modelo (GUILBERT 1988; TORRES \& KAREL 1985; VODJANI \& TORRES 1990).

Los primeros estudios de aplicación de antimicrobianos y fungicidas fueron por inmersión de los alimentos en una solución del aditivo. Esta metodología, inicialmente, fue efectiva para reducir el número total de microorganismos viables, pero en condiciones de almacenamiento, el alimento se deteriora de la superficie. Una alternativa ha sido la aplicación de fungicidas en emulsiones a base de cera o en suspensión en agua sobre cítricos. Sin embargo, la emulsión es menos eficaz, quizá debido a la encapsulación del aditivo en la fase lipídica, adicionalmente, la eficiencia del aditivo depende también del método de aplicación (ECKER \& KOLBEZEN 1977). Otro material para la preparación de recubrimientos es el quitosán el cual ha sido ampliamente estudiado. Cuando se combina con hidroxipropil metilcelulosa y se aplica en fresa, puede controlar el crecimiento de Cladiosporium sp y Rhizopus sp (PARK et al., 2005).

Otro grupo de compuestos de alto interés para la conservación de alimentos son los antioxidantes (tales como compuestos fenólicos) ya que aumentan la estabilidad de los componentes de los alimentos, especialmente de los de origen lipídico, mantienen el valor nutrimental y color al prevenir la rancidez oxidativa, la degradación y la decoloración. Algunos otros ácidos como el cítrico y ascórbico son agentes quelantes de metales. Compuestos fenólicos como el hidroxianisol butilado $(\mathrm{BHA})$, hidroxitolueno butilado $(\mathrm{BHT})$, hidroxiquinona terciaria butilada (TBQH), galato de propilo y tocoferoles inhiben la oxidación de los lípidos. Este tipo de compuestos, entre muchos otros, pueden ser incorporados en recubrimientos comestibles y al retenerse en la superficie del alimento se vuelven más eficaces ya que la oxidación es un fenómeno aire-superficie. También, este tipo de recubrimientos pueden reducir la oxidación enzimática (NISPEROS-CARRIEDO et al., 1990).

\section{RESULTADOS Y DISCUSIÓN}

\subsection{FABRICACIÓN COMERCIAL DE PELÍCULAS COMESTIBLES CON ADITIVOS BIOACTIVOS}

La fabricación comercial de películas comestibles a base de polímeros usando métodos modernos de fundición y secado comenzó alrededor de 1960. La hidroxipropil metilcelulosa es un polímero grado alimenticio fabricado por la Dow Chemical Company que fue usado para fabricar películas comestibles. Esta película se realizó para la industria de la panificación con volúmenes pre-medidos de vitaminas y mezclas de enriquecimiento y esta aplicación permanece hasta la fecha. Dicha empresa (www.dow.com), tiene una gama de polímeros funcionales denominada "AMPLYFYTM Functional Polymers" con el slogan "Ofrecer una gama única de propiedades y rendimiento mejorado para la capitalización y modificación de polímeros, adhesivos, capas de unión y más". Sin embargo, muchas otras películas se han desarrollado a lo largo de los años empleando casi todos los polímeros comestibles conocidos. Los ésteres de celulosa, almidón, pectina, alginato, gelatina, pululano, y mezclas de los mismos se encuentran en uso comercial. Otros polímeros naturales y sintéticos, así como proteínas se usan en volúmenes más pequeños. Solo un pequeño número de empresas en Estados Unidos y el mundo han desarrollado y construido equipos e instalaciones para la producción de películas comestibles (Sandvik Process Systems, LLC, Berndorf Belt Systems, Inc., Faustel, Inc., Werner Mathis USA, Inc., C. A. Litzler Co., Inc., Extrusion Dies, Inc., New Era Converting Machine, Admix, Inc.) (ROSSMAN 2009).

Hoy en día, las aplicaciones comerciales de las películas comestibles siguen apareciendo 
a un ritmo tan rápido que no hay una lista actualizada y completa de cada producto en el que se aplica. Las mejoras en la formulación de los recubrimientos y métodos de fabricación contribuyen a ampliar la utilidad de los mismos. Sin embargo, la mayor parte de las aplicaciones se dividen en unas categorías generales (Tabla 4).

TABLA 4. TIPOS DE APLICACIONES DE RECUBRIMIENTOS

\begin{tabular}{|c|c|}
\hline Categorías & Ejemplos de aplicación \\
\hline Empacado & $\begin{array}{l}\text { Vitaminas, enzimas, colorantes alimenticios, aditivos } \\
\text { alimentarios, bebidas mezcladas, potenciadores. }\end{array}$ \\
\hline Películas libres & $\begin{array}{l}\text { Refrescantes de aliento, pasta de dientes, dulces, et- } \\
\text { iquetas, nutraceuticos, medicamentos de venta libre } \\
\text { (OTC), anticonceptivos. }\end{array}$ \\
\hline Wraps & $\begin{array}{l}\text { Vitaminas, curado de carne, sushi, esmaltes para } \\
\text { carne, mezclas de especias. }\end{array}$ \\
\hline
\end{tabular}

TABLA 5. LISTADO DE RECUBRIMIENTOS COMESTIBLES COMERCIALMENTE USADOS

\begin{tabular}{|c|c|c|}
\hline $\begin{array}{l}\text { Nombre } \\
\text { Freshseel }^{\mathrm{TM}}\end{array}$ & $\begin{array}{l}\text { Componente principal } \\
\text { Esteres de sacarosa }\end{array}$ & $\begin{array}{l}\text { Usos } \\
\text { Prolonga la vida de anaquel de } \\
\text { melón }\end{array}$ \\
\hline Fry Shield ${ }^{\mathrm{TM}}$ & Pectinato de calcio & $\begin{array}{l}\text { Reduce la absorción de grasa du- } \\
\text { rante el freído de pescado, papas, } \\
\text { y otros vegetales. }\end{array}$ \\
\hline Nature Seal ${ }^{\mathrm{TM}}$ & Ascorbato de calcio & $\begin{array}{l}\text { Manzana, aguacate, zanahoria, y } \\
\text { otros vegetales. }\end{array}$ \\
\hline Nutrasave $^{\mathrm{TM}}$ & $N$, O-Carboximetil quitosano & $\begin{array}{l}\text { Reduce la pérdida de agua en } \\
\text { aguacate, retiene la firmeza. }\end{array}$ \\
\hline Seal gum, Spray gum ${ }^{\mathrm{TM}}$ & Acetato de calcio & $\begin{array}{l}\text { Previene el oscurecimiento de pa- } \\
\text { pas durante el freído. }\end{array}$ \\
\hline Semperfresh ${ }^{\mathrm{TM}}$ & Esteres de sacarosa & $\begin{array}{l}\text { Protege las frutas de hueso de la } \\
\text { perdida de agua y decoloración. }\end{array}$ \\
\hline
\end{tabular}

\section{CONCLUSIÓN}

La tecnología de películas y recubrimientos comestibles enriquecidos con compuestos bioactivos no es nueva, pero son necesarios más estudios para comprender la relación entre la atmosfera interna causada por el recubrimiento y la velocidad de los procesos fisiológicos como la respiración, ablandamiento de tejidos, reacciones metabólicas, producción de metabolitos y compuestos secundarios generados durante el almacenamiento. Comprender el comportamiento o el efecto causado por la adición de compuestos bioactivos que son capaces de prolongar la vida de anaquel requiere de intensa investigación. Hay muchos factores que deben ser considerados y 
estudiados para atribuir los efectos en el desempeño del recubrimiento para bienes específicos como la solución/emulsión y/o película (composición, concentración, viscosidad, densidad, propiedades mecánicas, de permeabilidad, térmicas, grosor, temperatura, condiciones atmosféricas, etc.) y el producto en el que se aplica (fresco o mínimamente procesado, tasa de respiración, actividad de agua, etc.). El objetivo final es generar recubrimientos que puedan ser comercializados y por ende, las investigaciones futuras deben ser interdisciplinarias para ofrecer información integral para diseñar recubrimientos específicos para productos específicos y amigables con el ambiente o "Tailor-made".

\section{REFERENCIAS}

1. ALI, A. et al.,. Gum arabic as a novel edible coating for enhancing shelflife and improving postharvest quality of tomato (Solanum lycopersicum L.) fruit. Postharvest Biology and Technology, v. 58, p. 42-47, 2010.

2. ALLEN, L. et al.,. Edible carbohydrate food coatings II. Evaluation of fresh meat products. Food Technology, v. 17, p. 1442-1448, 1963.

3. AYRANCY, E.; TUNC, S. The effect of edible coatings on water and vitamin C loss of apricots (Armeniaca vulgaris Lam.) and green peppers (Capsicum annuum L.). Food Chemistry, v. 87, p. 339-342, 2004.

4. BALDWIN, E. A. Edible coatings for fresh fruits and vegetables: past, present, and future. In: KROCHTA, J. M.;BALDWIN, E. A., et al (Ed.). Edible Coatings and Films to Improve Food Quality Lancaster, PA Technomic, 1994. p.25-64.

5. BALDWIN, E. A. et al.,. Effect of two edible coatings with different permeability characteristics on mango (Mangifera indica L.) ripening during storage. Postharvest Biology and Technology, v. 17, p. 215-226, 1999.

6. BALDWIN, E. A.; NISPEROS-CARRIEDO, M. O.; BAKER, R. A. Edible coatings for lightly processed fruits and vegetables. HortScience, v. 30, n. 1, p. 35-40, 1995.

7. BALDWIN, E. A.; NISPEROS-CARRIEDO, M. O.; BAKER, R. A. Use of edible coatings to preserve quality of lightly (and slightly) processed products. Critical Reviews in Food Science and Nutrition, v. 36, n. 6, p. 509-524, 1995.

8. BALDWIN, E. A.; WOOD, B. Use of edible coating to preserve pecans at room temperature. HortScience, v. 41, n. 41, p. 1, 2006.

9. BANKS, N.; DADZIE, B.; CLELAND, D. Reducing gas exchange of fruits with surface coatings. Postharvest Biology and Technology, v. 3, p. 269-284, 1993.

10. CARLIN, F. et al.,. Utilization of zein coating and sorbic acid to reduce Listeria monocytogenes growth on cooked sweet corn. Journal of Food Science, v. 66, n. 9, p. 1385-1389, 2001.

11. CERTEL, M.; USLU, M. K.; OZEDEMIR, F. Effects of sodium caseinate- and milk protein concentrate based edible coatings on the postharvest quality of Bing cherries. Journal of the Science of Food and Agriculture, v. 84, n. 10, p. $1229-1234,2004$.

12. CHIUMARELLI, M. et al.,. Fresh cut 'Tommy Atkins' mango pre-treated with citric acid and coated with cassava (Manihot esculenta Crantz) starch or sodium alginate. Innovative Food Science and Emerging Technologies, 2011.

13. CLAYPOOL, L. L. The waxing of deciduous fruits. American Society of Horticultural Science Proceedings, v. 37 , p. $443-447,2004$.

14. COSLER, H. B. Methods of producing zein coated confectionery. US 2791509 A 1957.

15. CURTIS, G. J. Some experiments with edible coatings on the long-term storage of citrus fruits. Proceedings of the Sixth International Citrus Congress, v. 3, p. $1514-1520,1988$.

16. DEELL, J. R.; PRANGE, R. K.; PEPPELENBOS, H. W. Postharvest physiology of fresh fruits and vegetables. In: CHAKRAVERTY, A.;JUJUMDAR, A. S., et al (Ed.). Handbook of Postharvest Technology. New York: Marcel Dekker, 2003. p. $455-484$

17. DOMENICO, J. A.; RAHMAN, A. R.; WESTCOTT, D. E. Effects of fungicides in combination with hot water and wax on the shelf life of tomato fruit. Journal of Food Science, v. 37, p. $957-960,1972$.

18. DONHOWE, I. G.; FENNEMA, O. The effects of plasticizers on crystallinity, permeability, and mechanical-properties of methylcellulose films. Journal of Food Processing and Preservation, v. 17, p. 247 - 257, 1993.

19. EAKS, I. L.; LUDI, W. A. Effects of temperature, washing and waxing on the composition of internal atmosphere of orange fruits. Proceedings of the American Society for Horticultural Science, v. 76, p. $220-228,1960$.

20. ECKER, J. W.; KOLBEZEN, M. J. Influence of formulation and application method on the effectiveness of benzimidazole fungicides for controlling postharvest diseases of citrus fruit. Netherlands Journal of Plant Pathology, v. 83, p. 343-352, 1977. 
21. FEENEY, R. D.; HARARALAMPU, S. G.; GROSS, A. Method of collating food with edible oil barrier film and product thereof. 5, 126-152 1992.

22. GHAOUTH, A. E. et al.,. Chitosan coating effect on storability and quality fresh strawberries. Journal of Food Science, v. 56, n. 6, p. $1618-1631,1991$.

23. GONZÁLEZ-AGUILAR, G. A.; ZAVALETA-GATICA, R.; TIZNADO-HERNÁNDEZ, M. E. Improving postharvest quality of mango 'Haden' by UV-C treatment. Postharvest Biology and Technology, v. 45, p. 108-116, 2007.

24. GUILBERT, S. Use of superficial edible layer to protect intermediate moisture foods: Application to the protection of tropical fruit dehydrated by osmosis. In: SEOW, C. C. (Ed.). Food Preservation by Moisture Control. London: Elsevier Applied Science Publishers Ltd., 1988. p.119-219

25. HAGENMAIER, R. D. Evaluation of a polyethylene-candelilla coating for 'Valencia' oranges. Postharvest Biology and Technology, v. 19, p. 147-154, 2000.

26. HAGENMAIER, R. D.; SHAW, P. E. Gas-permeability of fruit coating waxes. Journal of the American Society for Horticultural Science, v. 117, n. 1, p. $105-109,1992$.

27. HALLMAN, G. J. et al.,. Mortality of Carribean fruit fly (Diptera: Tephritidae) immatures in coated fruits. Journal of Economic Entomology, v. 87, p. 752 - 757, 1994.

28. HARDENBURG, R. E. Wax and related coatings for horticultural products. A bibliography. Agricultural Research Service Bulletin, p. 51-15, 1967.

29. HAVARD, C.; HARMONY, M. X. Improved process for preserving meat, fowls, fish etc. 909441869.

30. HOFFMAN, A. F. Preserving Fruit. 191601041916.

31. ISMAIL, M. Cold treatment: a successful quarantine method for Florida grapefruit shipped to Japan. Packinghouse Newsletter, v. 158, p. 1-2, 1989.

32. KAYS, S. J.; PAULL, R. E. Stress in harvested products. In: (Ed.). Postharvest Biology. Athens, GA, : Exon Press, 2004. p.355- 414 .

33. LAZARUS, C. R. et al.,. Evaluation of calcium alginate coating and protective plastic wrapping for the control of lamb carcass shrinkage. Journal of Food Science, v. 41, p. 639-642, 1976.

34. LEE, S. Y.; KROCHTA, J. M. Accelerated shelf-life testing of whey-protein-coated peanuts analyzed by static headspace gas chromatography. Journal of Agricultural Food Chemistry, v. 50, n. 7, p. 2022-2028, 2002.

35. LIN, D.; ZHAO, Y. Innovations in the development and application of edible coatings for fresh and minimally processed fruits and vegetables. Comprehensive Reviews in Food Science and Food Safety, v. 6, p. 60 - 75, 2007.

36. MEI, Y. et al.,. Using edible coating to enhance nutritional and sensory qualities of baby carrots. Journal of Food Science, v. 67, p. $1964-1968,2002$.

37. MEYERS, M. A. Use of edible films to prolong chewing gum shelf-life. 52865021994.

38. MURATORE, G. et al.,. The influence of using biodegradable packaging films on the quality decay kinetic of plum tomato (PomodorinoDatterino). Journal of Food Engineering, v. 67, p. 393-399, 2005.

39. NELSON, K.; FENNEMA, O. R. Methylcellulose films to prevent lipid migration in confectionery products. Journal of Food Science, v. 56, n. 2, p. $504-508,1991$.

40. NISPEROS, M. O.; BALDWIN, E. A. Edible coatings for whole and minimally processed fruits and vegetables. Food Australia, v. 48, n. 1, p. $27-31,1996$.

41. NISPEROS-CARRIEDO, M. O.; SHAW, P. E.; BALDWIN, E. A. Changes in volatile flavor components of pineapple orange juice as influenced by the application of lipid and composite films. Journal of Agricultural Food Chemestry, $v$. 38, p. $1382-1387,1990$.

42. OCHOA, E. et al.,. Evaluation of a Candelilla Wax-Based Edible Coating to Prolong the Shelf-Life Quality and Safety of Apples. American Journal of Agricultural and Biological Sciences, v. 6, n. 1, p. 92-98, 2011.

43. OLIVAS, G. I.; BARBOSA-CÁNOVAS, G. V. Edible coatings for fresh cut fruits. Critical Reviews in Food Science and Nutrition, v. 45, p. $657-670,2005$.

44. OLIVAS, G. I.; MATTINSON, D. S.; BARBOSA-CÁNOVAS, G. V. Alginate coatings for preservation of minimally processed "Gala" apples. Postharvest Biology and Technology, v. 45, p. 89 - 96, 2007.

45. PLIVAS, G. I.; RODRIGUEZ, J. J.; BARBOSA-CÁNOVAS, G. V. Edible coatings composed of methylcellulose stearic acid, and additives to preserve quality of pear wedges. Journal of Food Processing and Preservation, v. 27, p. 299 $-320,2003$. 
46. PARK, H. J. Development of advanced edible coatings for fruits. Trends in Food Science and Technology, v. 10, p. $254-260,1999$

47. PARK, H. J.; CHINNAN, M. S. Properties of edible coatings for fruits and vegetables. American Society of Agricultural Engineers, v. 90, n. 6510, p. 19, 1990.

48. PARK, S. I. et al.,. Antifungal coatings on fresh strawberries (Fragaria $x$ ananassa) to control mold growth during cold storage. Journal of Food Science, v. 70, n. 4, p. $202-207,2005$.

49. PAULINKA, F. Quality consideration in the selection of confectionery fats. . The Manufacturing Confectionerie, p. 7581, 1986.

50. PAVLATH, A. E.; ORTS, W. Edible films and coatings: Why, What, and How? Edible films and coatings for food applications, v. 1, p. 1-23, 2009.

51. PÉREZ-GAGO, M. B.; ROJAS, C.; DEL RÍO, M. A. Effect of hydroxypropyl methylcellulose-lipid edible composite coatings on plum (cv. Autumn giant) quality during storage. Journal of Food Science, v. 68, n. 3, p. 879-883, 2003.

52. PÉREZ-GAGO, M. B.; SERRA, M.; DEL RÍO, M. A. Color change of fresh-cut apples coated with whey protein concentrate-based edible coatings. Postharvest Biology and Technology, v. 39, p. 84 - 92, 2006.

53. PLATENIUS, H. Wax Emulsions for Vegetables. Cornell University Agricultural Experimental Station Bulletin, v. 43, p. 723-743, 1939.

54. POLANSKI, S. Frying food with reduced frying medium uptake - by cooking food to remove water applying coating of swollen dispersion containing natural edible polymer, drying and frying. 5 232-721 1993.

55. QUEZADA-GALLO, J. et al.,. Biopolymers used as edible coating to limit water transfer, colour degradation and aroma compound 2-pentanone lost in Mexican fruits. Acta Horticulturae, v. 682, n. 3, p. 1709 - 1716, 2005.

56. RADINA, P. M.; ECKERT, J. W. Evaluation of imazilil efficacy in relation to fungicide formulation and wax formulation. In: COHEN, R. e MENDEL, K. (Ed.). Citriculture proceedings of the sixth international citrus congress. Philadelphia PA: Balaban Publishers, 1988. p.1427 - 1434.

57. RIBEIRO, C. et al.,. Optimization of edible coating composition to retard strawberry fruit senescence. Postharvest Biology and Technology, v. 44, p. $63-70,2007$.

58. ROSSMAN, J. M. Commercial manufacture of edible films. Edible films and coatings for food applications, v. 14, p. 367-390, 2009.

59. SAUCEDO-POMPA, S. et al.,. Effect of candelilla wax with natural antioxidants on the shelf life quality of fresh-cut fruits. Journal of Food Quality, v. 30, p. 823-836, 2007.

60. SAUCEDO-POMPA, S. et al.,. Edible film based on candelilla wax to improve the shelf life and quality of avocado. Food Research International, v. 42, p. 511-515, 2009.

61. SHAW, C. P.; SECRIST, J. L.; TUOMY, J. M. Method of extending the storage life in the frozen state of precooked foods and products thereof. 41962191980.

62. SOTHORNVIT, R.; RODSAMRAN, P. Effect of a mango film on quality of whole and minimally processed mangoes. Postharvest Biology and Technology, v. 47, p. 407-415, 2008.

63. TAY, S. L.; PERERA, C. O. Effect of 1-methylcyclopropene treatment and edible coatings on the quality of minimally processed lettuce. Journal of Food Science, v. 69, n. 2, p. 131-135, 2004.

64. TORRES, J. A.; KAREL, M. Microbial stabilization of intermediate moisture food surfaces. III. Effect of surface preservative concentration and surface $\mathrm{pH}$ control on microbial stability of an intermediate moisture cheese analog. Journal of Food Process and Preservation, v. 9, p. $107-119,1985$.

65. VARGAS, M. et al.,. Quality of cold-stored strawberries as affected by chitosan-oleic acid edible coatings. Postharvest Biology and Technology, v. 41, p. 164-171, 2006.

66. VODJANI, F.; TORRES, J. A. Potassium sorbate permeability of methylcellulose and hydroxypropyl methylcellulose coatings: Effect of fatty acids. Journal of Food Science, v. 55, n. 3, p. $841-846,1990$.

67. WELLS, J. M. Heated wax-emulsion with benomyl and 2,6-dichloro-4-nitroaniline for control of postharvest decay of peaches and nectarines. Phytopathology, v. 62, p. $129-133,1971$.

68. WILLIAMS, S. K.; OBLINGER, J. L.; WEST, R. L. Evaluation of calcium alginate film for use on beef cuts. Journal of Food Science, v. 43, p. $292-301,1978$.

69. WONG, D. W. S.; CAMIRAND, W. M.; PAVLATH, A. E. Development of edible coatings for minimally processed fruits and vegetable. In: KROCHTA, J. M.;BALDWIN, E. A., et al (Ed.). Edible Coatings and Films to Improve Food Quality. Lancaster, P.A.: Technomic, 1994. p.65-88. 


\section{AGRADECIMIENTOS}

Los autores agradecen el apoyo financiero brindado por el Consejo Nacional de Ciencia y Tecnología (CONACYT), a la Dra. Blanca Valdivia Urdiales por el apoyo brindado en la escritura de este manuscrito y al M.C. Jorge Alejandro Aguirre Joya por la edición del mismo. 